\title{
Efficacy of complementary and integrative medicine on health-related quality of life in cancer patients: a systematic review and meta-analysis
}

This article was published in the following Dove Press journal: Cancer Management and Research

\author{
Wan-Fu Lin ${ }^{1} * *$ \\ Mao-Feng Zhong ${ }^{2, *}$ \\ Qing-Hui Zhou' \\ Yu-Ren Zhang' \\ Huan Wang' \\ Zhi-Hao Zhao' \\ Bin-Bin Cheng' \\ Chang-Quan Ling' \\ 'Department of Traditional Chinese \\ Medicine, Changhai Hospital, Naval \\ Medical University, Shanghai 200433, \\ People's Republic of China; ${ }^{2}$ Graduate \\ School of Shanghai University of Chinese \\ Medicine, Shanghai 20I203, People's \\ Republic of China
}

*These authors contributed equally to this work
Correspondence: Bin-Bin Cheng; Chang-Quan Ling

Department of Traditional Chinese Medicine, Changhai Hospital, Naval Medical University, 168 Changhai Road, Shanghai 200433, People's Republic of China

Tel +86 213 II6 1966; +86 218 I87 I559

Fax +862181871559

Email cbb8202@126.com;

changquanling@smmu.edu.cn

\begin{abstract}
Complementary and integrative medicine (CIM) has been used for improving health-related quality of life (HRQOL) in patients with cancer. The objective of this review is to evaluate the effects of CIMs on the HRQOL of cancer patients. We identified randomized controlled trials (RCTs) involving patients with cancer at any stage by retrieving electronic databases from the inception to February 14, 2018 (Systematic Review Registration: PROSPERO CRD42018091609). The main outcomes were HRQOL scores and related domains such as physical well-being scores. The standardized mean difference was used for the analysis and heterogeneity was assessed with the $I^{2}$ statistic. A Bayesian framework was used to estimate the ranking order of efficacy in HRQOL change. Finally, 34 RCTs with 3,010 patients were included. As a whole, the results showed clearly superior efficacy of CIM in improving HRQOL. For different domains of HRQOL, different CIM interventions may play different roles. The ranking order of efficacy in change HRQOL was qigong plus mindfulness, Chinese herbal medicine, multimodal complementary medicine, qigong, nutritional supplement, mindfulness, acupuncture, yoga, and massage, and it was different among different domains. There was no evidence of publication bias. In conclusion, CIM may improve the HRQOL of cancer patients. More studies, especially focusing on male cancer patients, are needed to increase the confidence level of our findings.
\end{abstract}

Keywords: complementary medicine, alternative medicine, integrative medicine, healthrelated quality of life, randomized controlled trials

\section{Introduction}

Data from GLOBOCAN 2012, produced by the International Agency for Research on Cancer, indicated that an estimated 14.1 million new cancer cases and 8.2 million cancer deaths occurred in 2012 worldwide. ${ }^{1}$ This trend has not been curbed by the progress of medical research.

Because low health-related quality of life (HRQOL), especially resulting from inadequate treatment (eg, chemotherapy), may deteriorate cancer patients' condition and even increase mortality, ${ }^{2}$ the HRQOL is a central consideration for many physicians in their decision-making process in catering to different treatment options. $^{3,4}$ Identifying the efficacy of long-term treatment strategies in improving low HRQOL in patients with cancer is of paramount importance.

Complementary and integrative medicine (CIM), according to the National Center for Complementary and Integrative Health, refers to the non-mainstream therapies which can be used along with conventional treatment. In general, it 
encompasses many diverse therapies including natural products (such as herbs and botanicals), mind and body practices (such as acupuncture, massage and mediation), and other complementary health approaches. ${ }^{5}$ With more interest in CIM and a growing body of evidence supporting the use of CIM among patients with cancer, more medical clinics and cancer centers are trying to address public interest and demand by providing CIM services. ${ }^{6-8}$ Unfortunately, many integrative practices remain under study, with insufficient evidence to be definitively recommended. The best CIM method for cancer patients has not yet been established. Therefore, in the present systematic review, we examined the randomized clinical trial (RCT) evidence to compare the relative efficacy of different CIM interventions, hoping to provide significant information for patients, health-care practitioners, and policy makers on the course of tumor treatment prescription to treat low HRQOL in patients with cancer.

\section{Materials and methods}

We followed the PRISMA guidelines for this systematic review and meta-analysis. ${ }^{9}$ A previously established protocol registered with PROSPERO (CRD42018091609) was conducted and associations of each CIM with HRQOL were compared using a direct meta-analysis and Bayesian network meta-analysis. Good research practices on indirect treatment comparisons, as emphasized in the International Society for Pharmacoeconomics and Outcomes Research Task Force, were rigorously followed $^{10,11}$ and quality of evidence was appraised by the Grading of Recommendations Assessment, Development and Evaluation (GRADE) criteria. ${ }^{12}$

\section{Search strategy and selection criteria}

We searched PubMed, MEDLINE, Embase, Web of Science, Cochrane Central, and Clinical Trial registries (http://www.clinicaltrials.gov and http://www.clinicaltrials register.eu) from inception to February 14, 2018. We also manually screened published systematic reviews and presentations from major conference proceedings such as the American Society of Clinical Oncology for additional studies. The references of the final included articles were also reviewed. The search was conducted by two investigators (MFZ and WFL) independently.

The search terms were "CIM", "complementary and integrative medicine", "complementary and alternative therapies", "complementary medicine", "alternative medicine", "integrative medicine", "HRQOL", "quality of life", "health related quality of life", "life quality", "neoplasia", "tumor", "cancer", "malignant neoplasm", "oncology", "onco*", and "integrative oncology".

To be eligible, RCTs comparing CIM-based intervention with a control group receiving no intervention for psychological functioning and HRQOL in patients with cancer were included. In addition, selected evaluation tools for overall HRQOL were those HRQOL questionnaires which were most widely used in clinical research, including the Functional Assessment of Cancer Therapy (FACT), ${ }^{13}$ Functional Assessment of Chronic Illness Therapy (FACIT), ${ }^{14}$ MD Anderson Symptom Inventory (MDASI), ${ }^{15}$ 36-item Short Form Health Survey 36 (SF$36),{ }^{16}$ and European Organisation for Research and Treatment of Cancer (EORTC). ${ }^{17}$

We excluded observational studies, trials with unclear effective CIM treatments (eg, music therapy and aromatherapy), and studies conducted in special populations (eg, patients with mental illness or care), to avoid excessive heterogeneity.

\section{Data abstraction and quality assessment}

Data from the included studies were extracted by two authors (YRZ and HW) independently on to a standardized form including the name of study, first author, study design, and blinding; patient characteristics; and the frequency, duration, and schedule of the primary intervention. The primary outcome was the HRQOL score changes between baseline and after treatment, which were measured in terms of several multidimensional generic questionnaires consisting of multiple domains such as physical well-being, social well-being, emotional wellbeing, sleep quality, and fatigue. All data were abstracted using study-reported modified intention-to-treat analysis. Data abstraction discrepancies were resolved by consensus in consultation with a third reviewer (ZHZ). The risk of bias of an individual study was assessed in the context of the primary outcome using the Cochrane Risk of Bias assessment tool. ${ }^{18}$

\section{Quality of evidence}

We assessed the quality of evidence of estimates derived from network meta-analysis using the GRADE methodology. ${ }^{12,19}$ For direct comparisons in this system, RCTs start at high quality and may be downgraded to levels of moderate, low, and very low quality owing to heterogeneity, risk of bias, indirectness, imprecision, and/ or publication bias. For the indirect estimates, it starts at 
the lowest rating of the two pairwise estimates that contribute as first-order loops, but may be further downgraded in consideration of imprecision or intransitivity (heterogeneity such as different clinical or methodological characteristics). The higher rating of the direct or indirect estimates would be applied to the network meta-analysis if their ratings were similar.

\section{Statistical analysis}

The DerSimonian and Laird random-effects model was used for direct meta-analysis to estimate pooled standardized mean differences (SMDs) and 95\% CI incorporating within- and between-study heterogeneity. ${ }^{20}$ The $I^{2}$ statistic was calculated to assess study heterogeneity. ${ }^{21}$ The Hartung-Knapp method was used to address possible type I errors in post-hoc sensitivity analyses. ${ }^{22}$ Funnelplot symmetry and Egger's regression test were used to assess the publication bias, with the test value $P<0.05$ indicating publication bias. ${ }^{23}$

For the indirect meta-analysis, we performed a random-effects network meta-analysis in ADDIS version 1.14.1. Network meta-analysis models in ADDIS are implemented in the Bayesian framework and estimated using Markov chain Monte Carlo (MCMC) methods. ${ }^{24}$ This approach is recommended by the National Institute for Health and Care Excellence (NICE) Decision Support Unit technical support documents on evidence synthesis. ${ }^{25}$ Since this network meta-analysis is an indirect comparison based on the comparison of placebo/conventional care without CIM and multiple CIMs, statistical analysis is performed directly under the consistency model without the need to carry out consistent tests. Statistical significance was assessed using 95\% CI, with CI spanning 1 indicating $P>0.05$, suggesting no statistical significance. Then, a network diagram was drawn and finally a rankorder graph of each CIM was constructed.

\section{Results}

\section{Characteristics of the included studies}

In total, 574 unique studies were found using the search strategy, most of which were duplicate records or not reporting RCTs. Thus, 149 full-text articles were fully reviewed according to the inclusion and exclusion criteria, resulting in a final sample of 34 studies (Figure 1). All studies are two-arm trials, in which one arm is a CIM intervention, including yoga (eight trials), nutritional supplement (NS) (six trials), Chinese herbal medicine $(\mathrm{CH})$ (four trials), acupuncture (four trials), multimodal complementary medicine (MCM) (three trials), qigong (three trials), mindfulness (MM) (three trials), massage (two trials), or qigong plus MM (one trial), while the other arm is placebo or usual care without CIM treatment.

The characteristics of patients included in the RCTs enrolled in this review are summarized in Table 1. Overall, these 34 trials were reported between 2006 and 2017 and included 3,010 participants (the range of size of trials was 13 to 275 participants). The primary outcome (HRQOL score changes) was reported in all studies. Among the trials, 16 trials were from the USA, five trials from Germany, four trials from China, four trials from Australia, two trials from Japan, and two trials from the UK; and South Korea, Malaysia, Turkey, and Italy each had one trial. The age of patients ranged from 44.7 to 70.3 years (median 56 years) across all studies, and 92\% were female. Breast cancer (20 studies) was the most studied cancer among the enrolled studies, followed by various cancers (seven studies), colorectal cancer (three studies), prostate cancer (one study), lung cancer (one study), hepatic carcinoma (one study), and ovarian cancer (one study). The mean HRQOL score of patients at the baseline of CIM treatment was 82.5 (range 20.7-152.1), while it was 80.4 (range 16.6-143.2) in the control group. However, after treatment, the mean HRQOL score of patients in the CIM group was 87 (range 24.4-145.2), while it was 81.8 (range 20-131.4) in the control group.

\section{Quality assessment and risk of bias of the included trials}

Using Cochrane's Risk of Bias assessment tool, the result indicated that 11 studies were scored as high quality. Most studies scored high risk are ascribed to the bias on blinding of personnel, since blinding of personnel was not applicable during the exercise interventions. Furthermore, several studies were judged as unclear risk of bias in random sequence generation, allocation concealment, blinding of participants and personnel, and blinding of outcome assessment. The results are shown in Figure 2.

\section{Direct meta-analysis of the included studies}

After extracting the data of the included studies, available direct comparisons and network of trials were compiled and are shown in Figure 3. All agents were associated with HRQOL and CIMs compared with placebo/usual care without CIMs. In post-hoc sensitivity analysis using the 


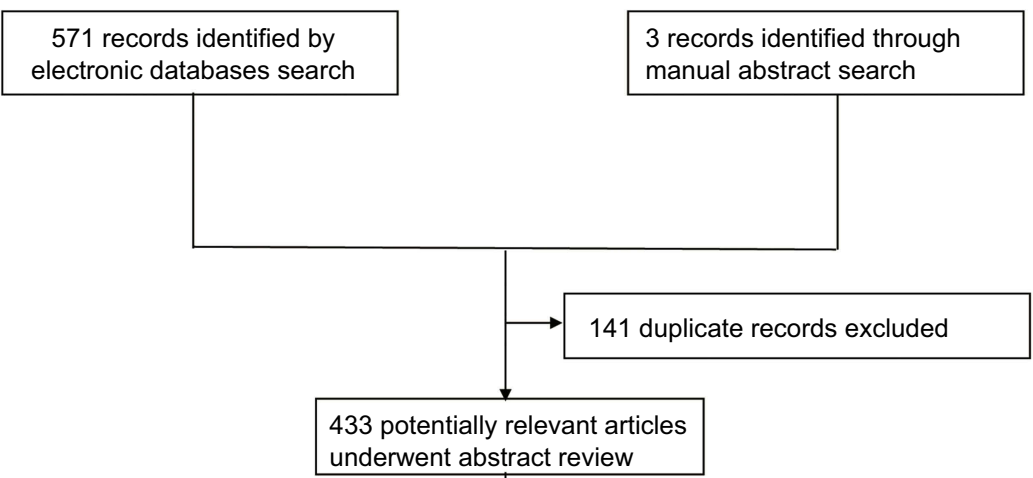

284 excluded

45 protocal or review articles

51 observational studies

46 unrelated to CIM interventions

17 population not of interest

78 outcome not of interest

47 no control group

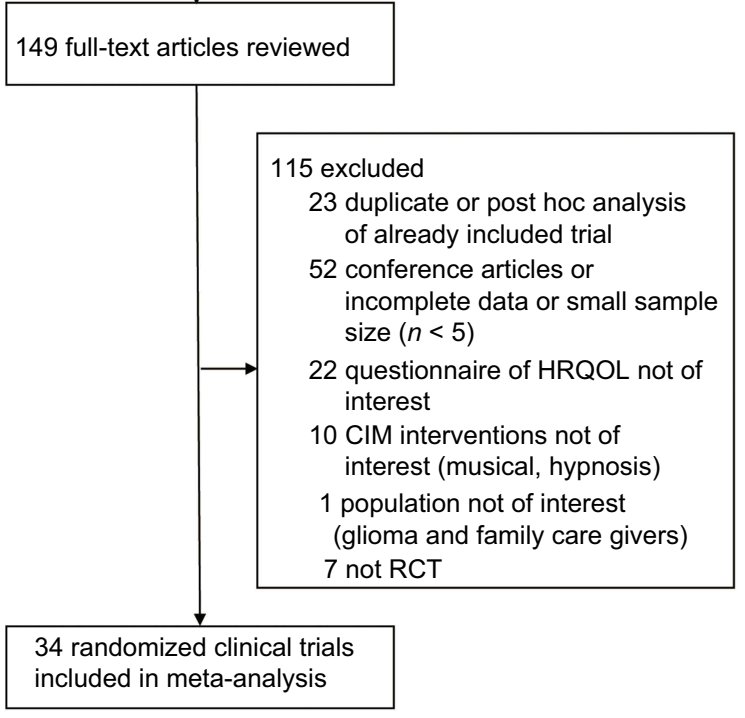

Figure I Flowchart of the study identification and selection process.

Abbreviations: CIM, complementary and integrative medicine; HRQOL, health-related quality of life; RCT, randomized clinical trial.

Hartung-Knapp method, all results were consistent. The results indicated that, taking usual care without CIM treatment as a comparator, all the CIM treatments reported the effects on HRQOL and emotional well-being. Most enrolled studies compared yoga and NS with control on all the treatment efficacy evaluation dimensions, such as HRQOL and emotional well-being. Furthermore, for the change in HRQOL from endpoint to baseline, we used direct metaanalysis and the results showed that all the subgroups of different CIM treatments did not show obvious heterogeneity. Therefore, a fixed-effect model was employed to test the effects. The test for total effect showed clearly superior efficacy of CIM treatments in improving HRQOL (mean difference 3.99 [2.32, 5.67]), although subgroup analysis demonstrated that only $\mathrm{CH}$ (mean difference $6.03[0.15$, 11.92]) and qigong + MM (mean difference 12.66 [8.75, 16.57]) was significantly favored over usual care (Figure 4). On the other hand, for the multiple domains related to HRQOL, the overall effect for total CIM treatments may improve emotional (SMD 0.18 [0.05, 0.31]) and physical well-being (SMD $0.22[0.06,0.37]$ ), with moderate heterogeneity (Table 2). Yoga seems to aggravate sleep quality (SMD $-0.81[-1.18,-0.08]$ ), which is contrary to the traditional conception that yoga may reduce sleep problems. ${ }^{60}$ 


\begin{tabular}{|c|c|c|c|c|c|c|c|c|c|}
\hline & $\begin{array}{l}\frac{\Xi}{\Xi} \\
\bar{\omega}\end{array}$ & $>$ & & & & & & & \\
\hline \multirow{5}{*}{ 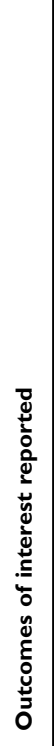 } & 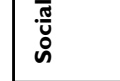 & 7 & $>$ & & $>$ & $>$ & 7 & & \\
\hline & 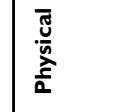 & $>$ & $>$ & & $>$ & $>$ & $>$ & & \\
\hline & 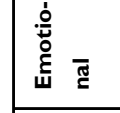 & $>$ & $>$ & & $>$ & $>$ & $>$ & & \\
\hline & 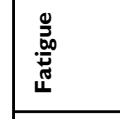 & $>$ & & & 7 & & $>$ & & \\
\hline & 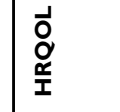 & $>$ & $>$ & $>$ & $>$ & $>$ & $>$ & $>$ & $>$ \\
\hline \multicolumn{2}{|c|}{ 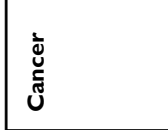 } & 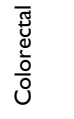 & 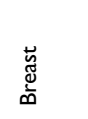 & 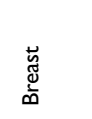 & 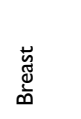 & 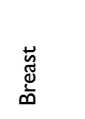 & 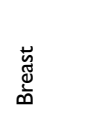 & 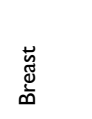 & 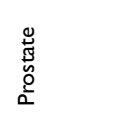 \\
\hline & 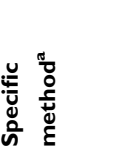 & 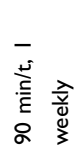 & $\begin{array}{ll}\bar{M} & \vec{y} \\
\bar{z} & \frac{\vec{y}}{3}\end{array}$ & 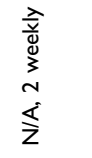 & 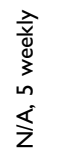 & 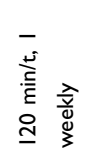 & 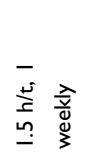 & 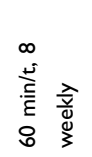 & 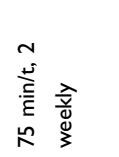 \\
\hline \multirow{4}{*}{ 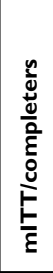 } & \multirow{2}{*}{ 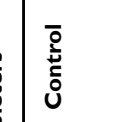 } & $\hat{N}$ & $\stackrel{m}{m}$ & $\tilde{\sim}$ & $\hat{N}$ & సి & F & 으 & $\stackrel{\infty}{\sim}$ \\
\hline & & $\bar{\lambda}$ & f & 7 & $\bar{m}$ & $\bar{m}$ & น̊ & $\underline{\underline{n}}$ & m \\
\hline & \multirow{2}{*}{ 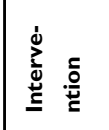 } & $\hat{\lambda}$ & $\bar{m}$ & 요 & $\hat{\lambda}$ & $\hat{\sim}$ & $\stackrel{ \pm}{\infty}$ & $=$ & $\pi$ \\
\hline & & $\hat{\sim}$ & f & g & $\tilde{m}$ & ஓे & $\stackrel{\infty}{\underline{0}}$ & $\underline{\underline{n}}$ & $\stackrel{m}{m}$ \\
\hline \multicolumn{2}{|c|}{ 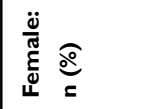 } & 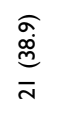 & $\underset{\text { s }}{\stackrel{\sigma}{\sigma}}$ & $\underset{m}{\stackrel{o}{g}}$ & 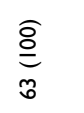 & $\frac{\overline{8}}{\frac{\sigma}{6}}$ & $\begin{array}{l}\stackrel{\widehat{\delta}}{\stackrel{\infty}{\sim}} \\
\stackrel{\sim}{\simeq}\end{array}$ & 总 & 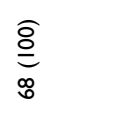 \\
\hline \multicolumn{2}{|c|}{ 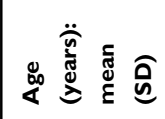 } & \multicolumn{8}{|c|}{ 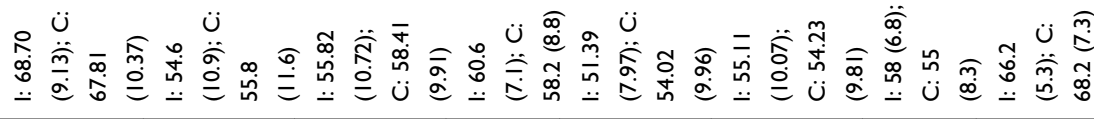 } \\
\hline \multicolumn{2}{|l|}{ 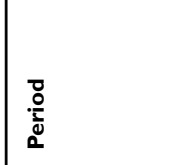 } & 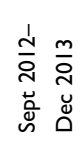 & 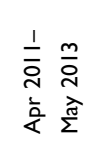 & 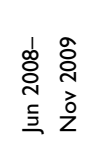 & 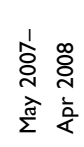 & 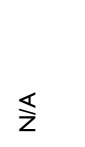 & 䓂 & 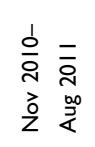 & 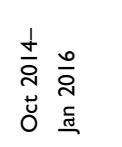 \\
\hline \multicolumn{2}{|l|}{ 売 } & 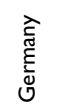 & כ & 胥 & 崩 & 离 & 㞻 & 离 & 㞻 \\
\hline \multicolumn{2}{|l|}{ ્ֻઁ } & $\stackrel{+}{\grave{N}}$ & $\stackrel{n}{\bar{N}}$ & $\stackrel{m}{\bar{N}}$ & $\overline{\mathrm{N}}$ & $\stackrel{\circ}{\circ}$ & ఫ్రి & $\frac{\sim}{\grave{n}}$ & $\hat{\bar{N}}$ \\
\hline \multicolumn{2}{|l|}{ 害 } & 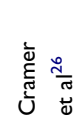 & 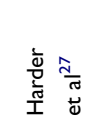 & 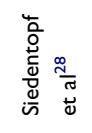 & 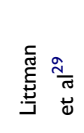 & 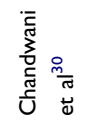 & 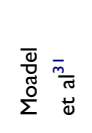 & 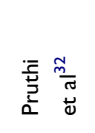 & 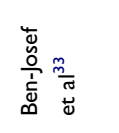 \\
\hline \multicolumn{2}{|l|}{$\sum_{U}^{\infty}$} & 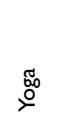 & & & & & & & \\
\hline
\end{tabular}




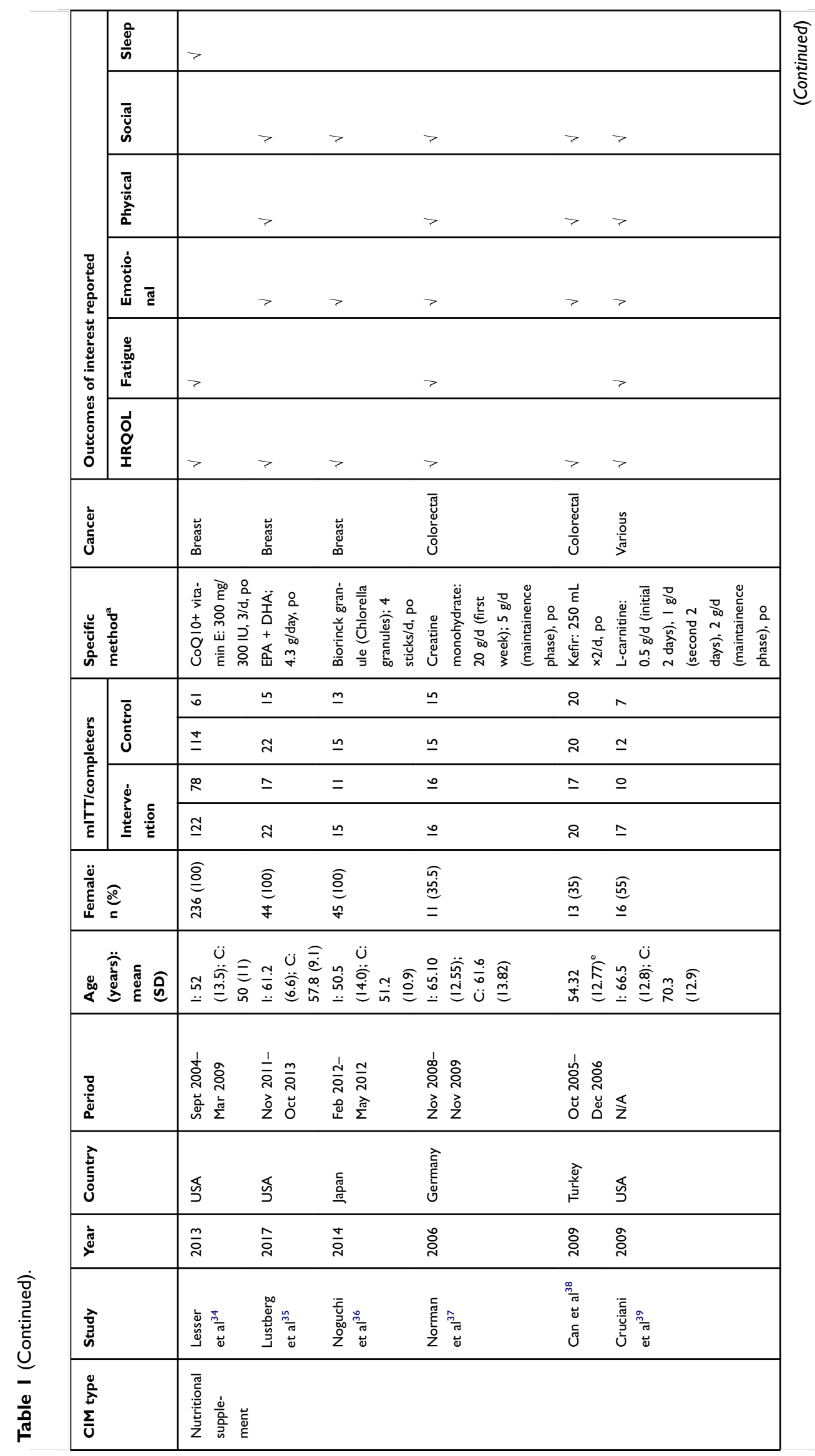




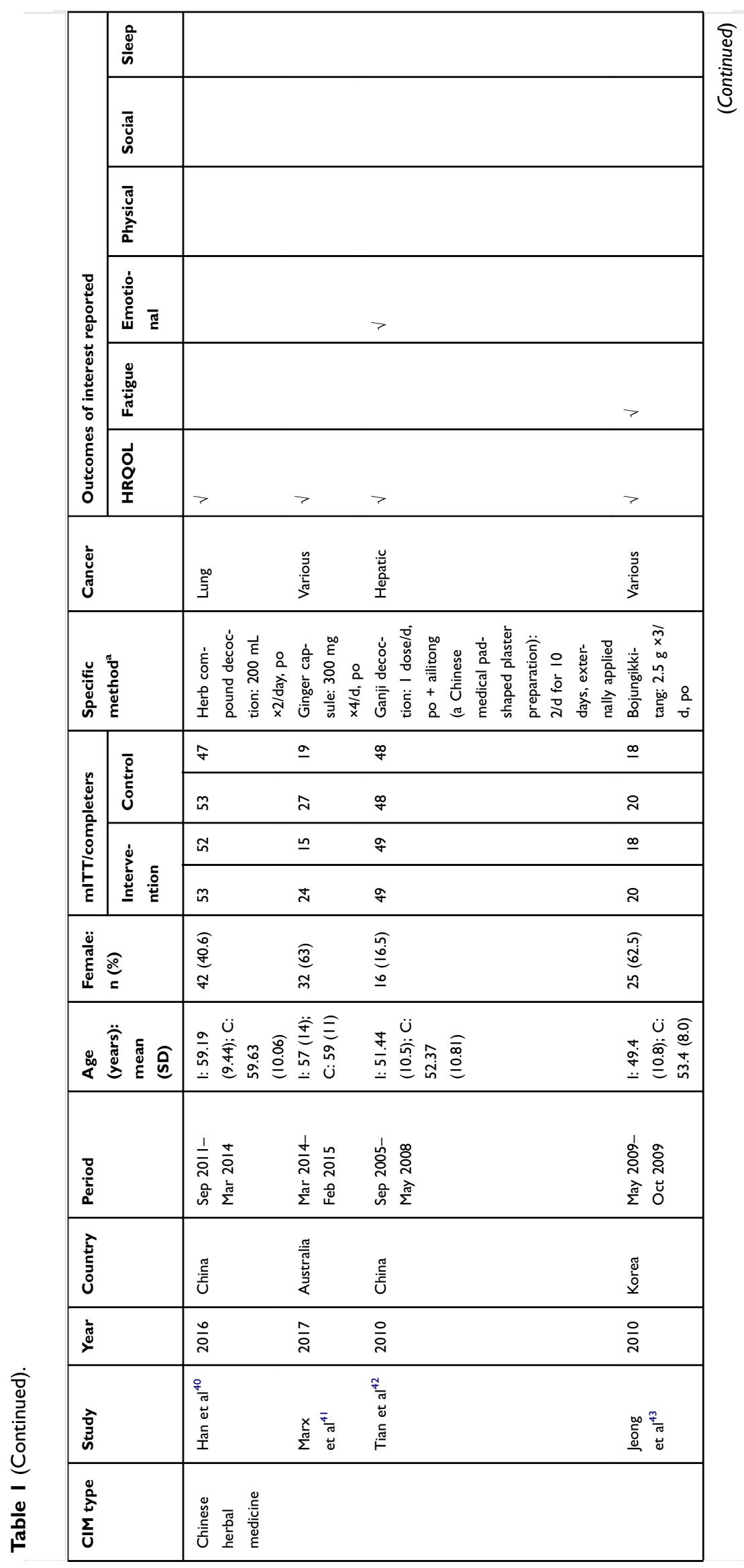




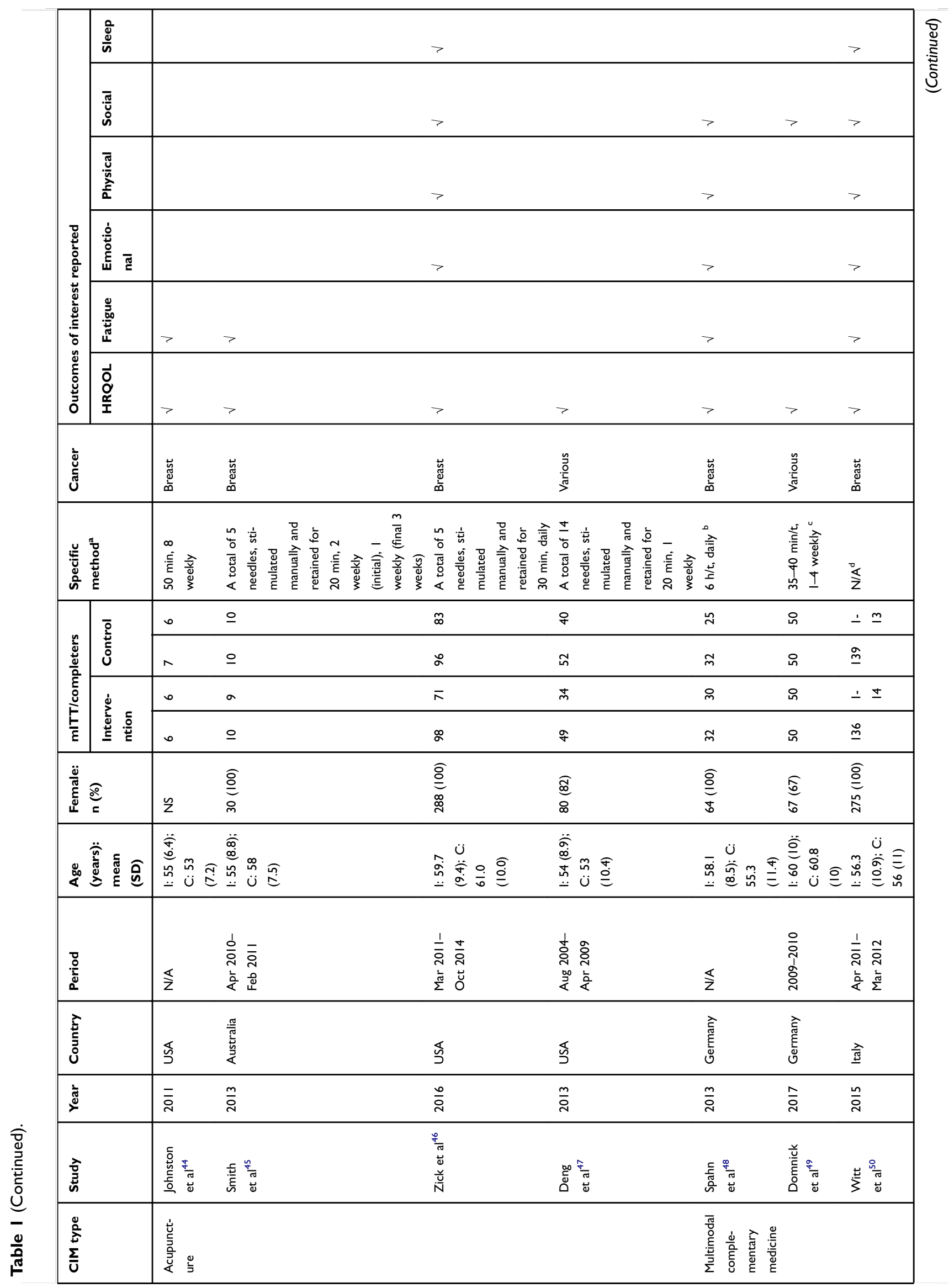




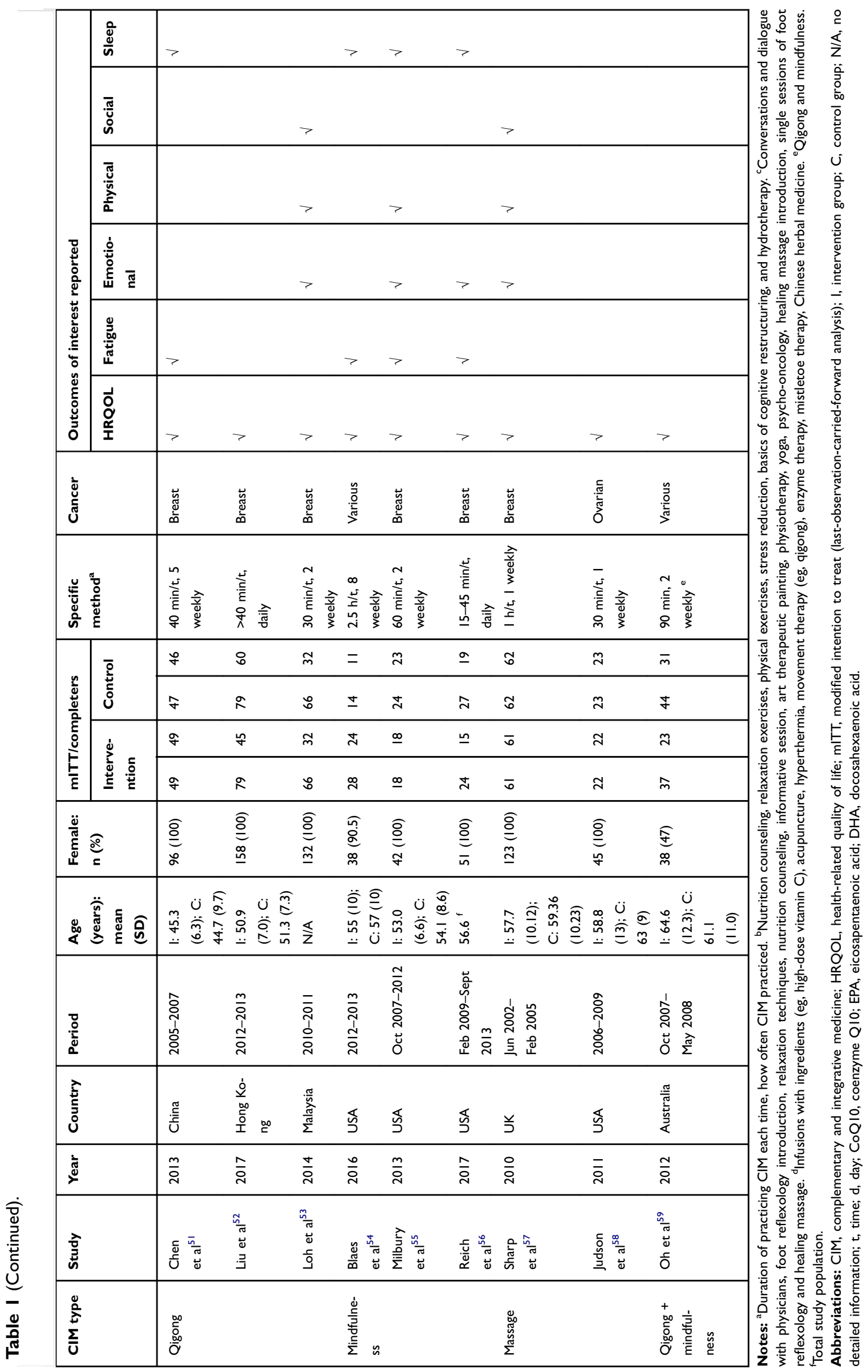


A

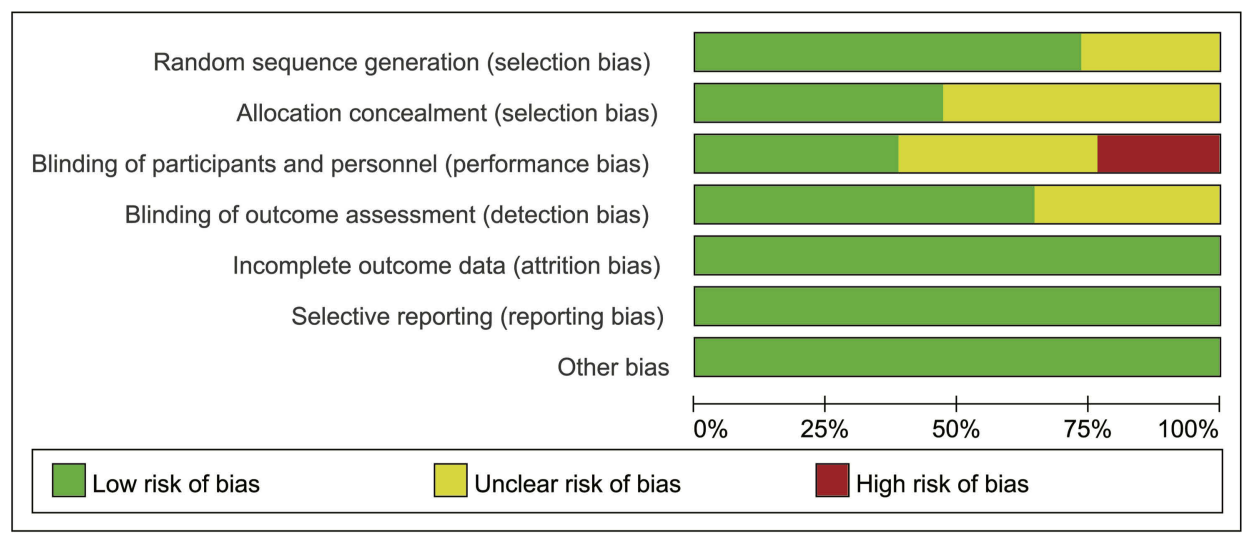

B

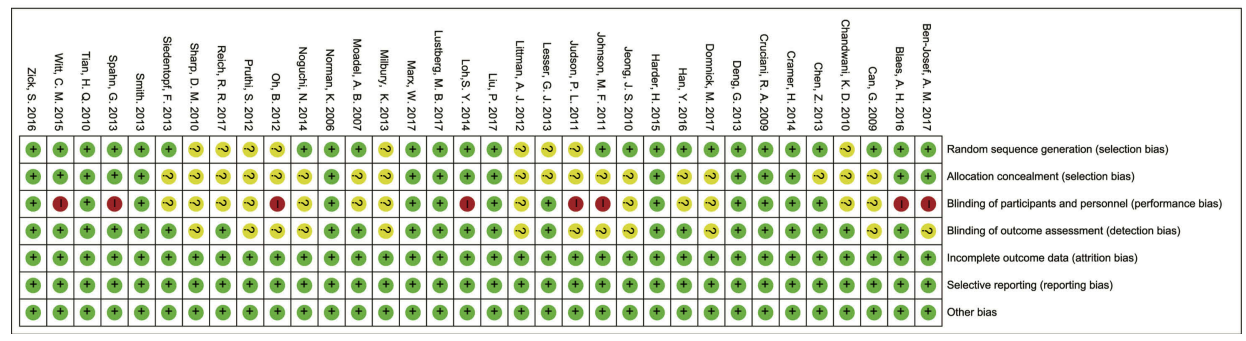

Figure 2 Quality assessment of the trials included in the analysis: review authors' judgments about each risk of bias item for all included studies (A), and for each included study (B).

A

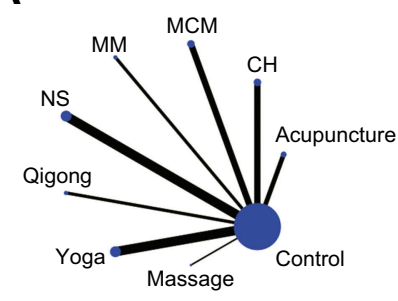

D

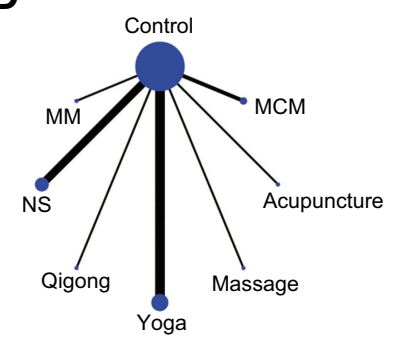

B

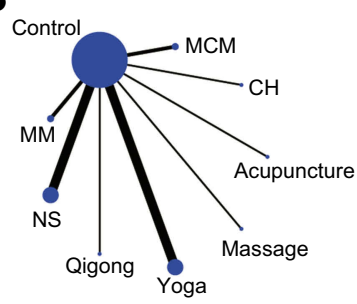

E

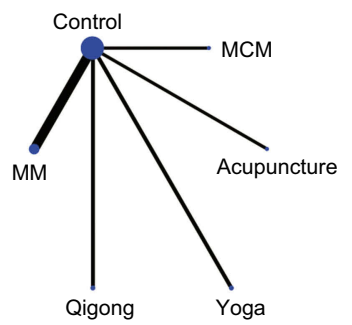

C

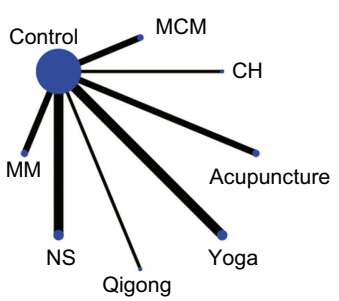

$\mathbf{F}$

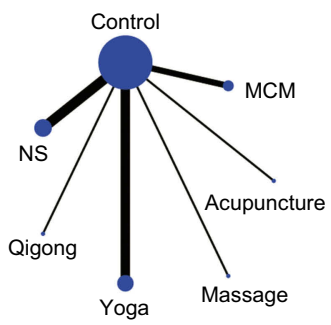

Figure 3 Network of included studies with the available direct comparisons for all outcomes. (A) Health-related quality of life (HRQOL); (B) emotional well-being; (C) fatigue; (D) physical well-being; (E) sleep quality; (F) social well-being. The size of the nodes and the thickness of the edges indicate the number of included studies.

Abbreviations: $\mathrm{CH}$, Chinese herbal medicine; MCM, multimodal complementary medicine; MM, mindfulness; NS, nutritional supplement.

\section{Network meta-analysis of the included studies}

To further demonstrate the relative effect of each intervention on the HRQOL, network meta-analysis was applied and the ranking probability for each treatment was estimated. Graphical results are shown in Figure 5. The overall ranks were interpreted by the surface under the cumulative ranking (SUCRA) technique. ${ }^{61}$ For HRQOL, 


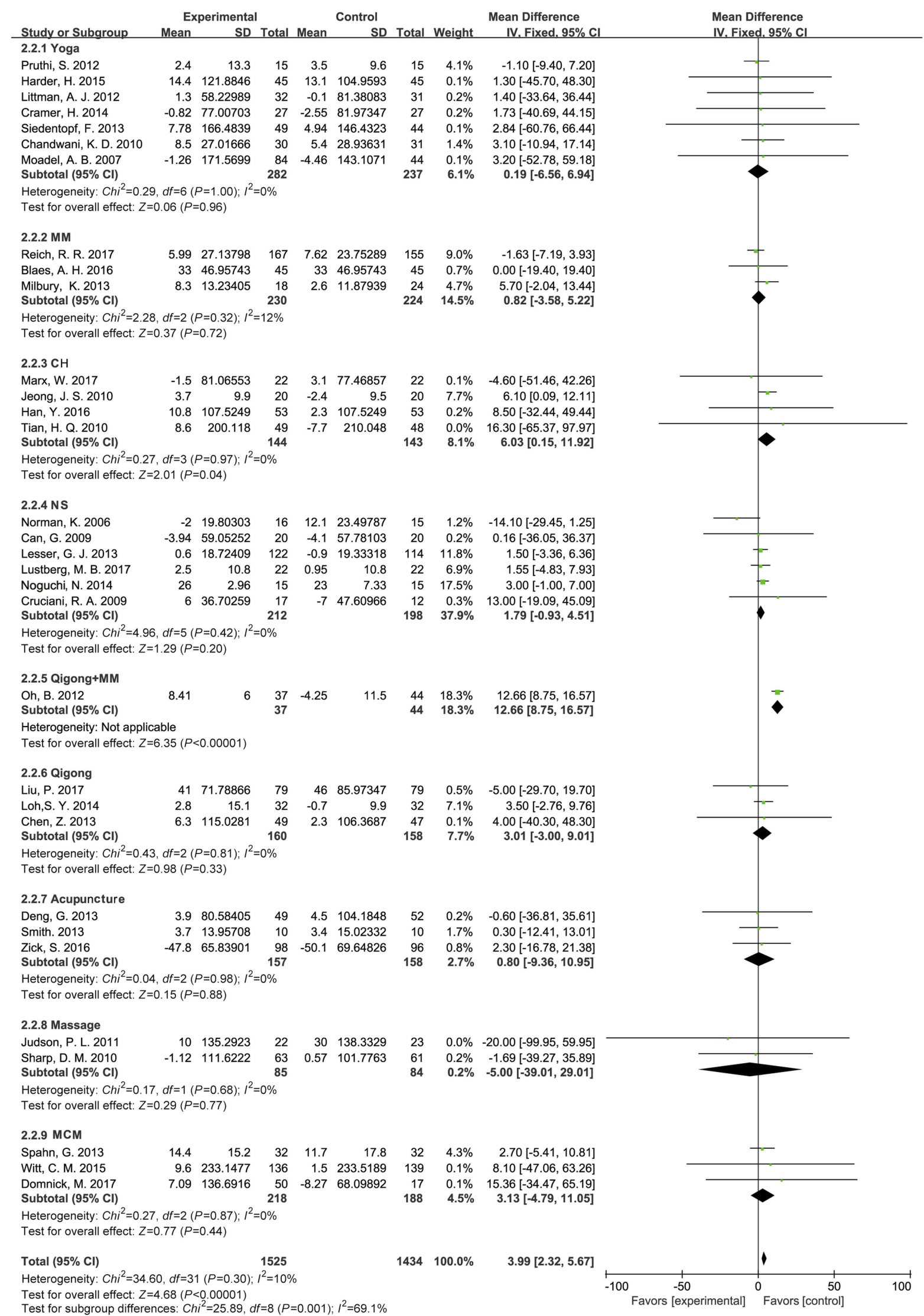

Figure 4 Direct meta-analysis of the change in health-related quality of life from endpoint to baseline.

Abbreviations: $\mathrm{CH}$, Chinese herbal medicine; MCM, multimodal complementary medicine; MM, mindfulness; NS, nutritional supplement. 


\begin{tabular}{|c|c|c|c|c|c|c|c|c|c|c|c|c|}
\hline & \multirow{3}{*}{ 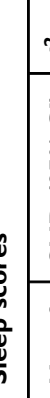 } & $\simeq$ & \multicolumn{3}{|c|}{ 今े $\stackrel{\circ}{\circ} \quad \mid$} & I & ○̊ & 1 & ঃ̊ & | & ○̊ & $\stackrel{\circ}{\stackrel{\circ}{\curvearrowright}}$ \\
\hline & & 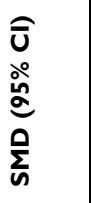 & 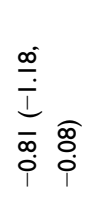 & 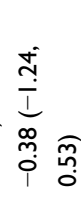 & & I & 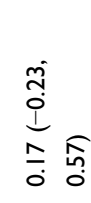 & I & 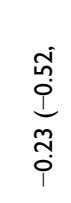 & & 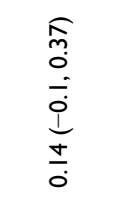 & $\begin{array}{l}\text { o } \\
\stackrel{0}{0} \\
\frac{1}{i} \\
\frac{\hat{m}}{i}\end{array}$ \\
\hline & & $\begin{array}{l}\frac{\mathscr{u}}{\tilde{z}} \\
\dot{z} \\
\dot{z}\end{array}$ & $\stackrel{-}{-}$ & 号 & 1 & I & $\bar{n}$ & I & f & I & $\stackrel{\circ}{n}$ & $r$ \\
\hline \multirow{3}{*}{\multicolumn{2}{|c|}{ 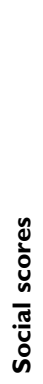 }} & $\simeq$ & ○ & I & | & ○ & ○̊ & 1 & ○̊ & ঃ̊ & 。 & ○̊ \\
\hline & & 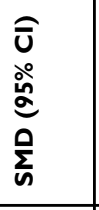 & 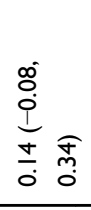 & I & I & $\begin{array}{l}\frac{\sigma}{0} \\
\bar{i} \\
\overline{\bar{i}}\end{array}$ & 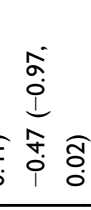 & I & & 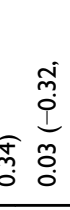 & 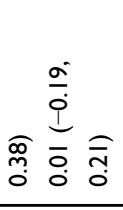 & 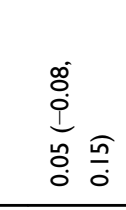 \\
\hline & & 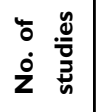 & 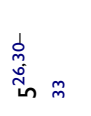 & & । & 总 & $\cong$ & I & \& & $\underline{n}$ & 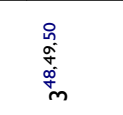 & $\underline{\varrho}$ \\
\hline \multirow{3}{*}{\multicolumn{2}{|c|}{ 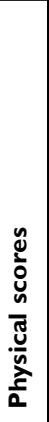 }} & $\simeq$ & $\begin{array}{l}\stackrel{\circ}{\mathrm{f}} \\
\stackrel{2}{R}\end{array}$ & ঃ̊ & । & $\frac{\circ}{m}$ & I & I & ○̊ & I & ○̊ & $\stackrel{\stackrel{\circ}{\infty}}{\stackrel{\rho}{\infty}}$ * \\
\hline & & $\begin{array}{l}\widehat{0} \\
\stackrel{\circ}{0} \\
\stackrel{0}{0} \\
\sum_{n}^{0}\end{array}$ & 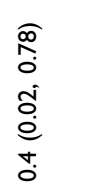 & 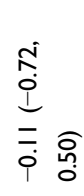 & I & 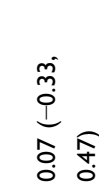 & 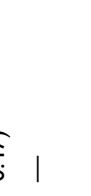 & I & $\begin{array}{l}\frac{0}{i} \\
\frac{1}{i}\end{array}$ & 厑 & $\begin{array}{l}\bar{f} \\
0 \\
0 \\
0 \\
0 \\
\frac{1}{0} \\
\frac{0}{0}\end{array}$ & 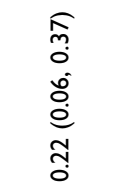 \\
\hline & & 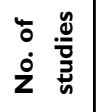 & 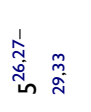 & $\stackrel{\circ}{n}$ & I & 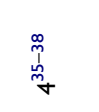 & 1 & 1 & f & I & $\underset{\substack{0 \\
\text { ond } \\
\sim}}{\sim}$ & $\underline{m}$ \\
\hline \multirow{3}{*}{\multicolumn{2}{|c|}{ 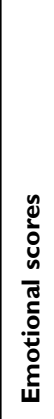 }} & $\simeq$ & ○े & ڤें & ○̊ & సัे & ○̊ & I & 今。 & ळ̊ & ○̊ & $\begin{array}{l}\stackrel{\circ}{\hat{N}} \\
\stackrel{m}{\circ}\end{array}$ \\
\hline & & 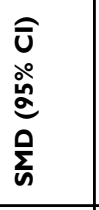 & 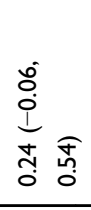 & 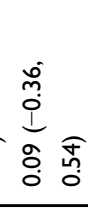 & 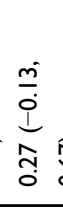 & E. & $=\frac{\substack{0 \\
0}}{\frac{m}{0}}$ & । & 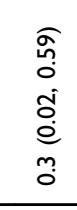 & $\frac{\frac{\infty}{0}}{\frac{\infty}{0}}$ & 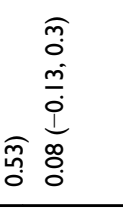 & 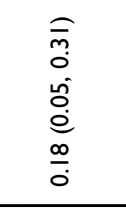 \\
\hline & & 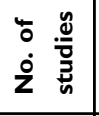 & 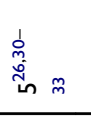 & 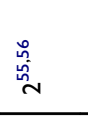 & i & 总 & $\stackrel{m}{-}$ & 1 & $\ddagger$ & in & 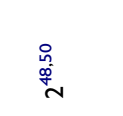 & $\underline{\infty}$ \\
\hline \multirow{3}{*}{\multicolumn{2}{|c|}{ 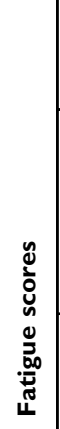 }} & $\simeq$ & ○ & 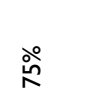 & ○̊ & $\stackrel{\circ}{\stackrel{2}{人}}$ & ○̊ & I & ○̊ & I & $\frac{\circ}{6}$ & $\begin{array}{l}\stackrel{\circ}{0} \\
\stackrel{0}{f}\end{array}$ \\
\hline & & 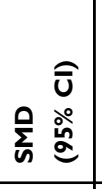 & 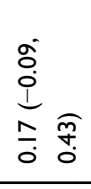 & 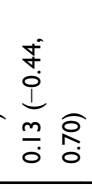 & 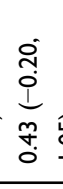 & 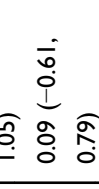 & 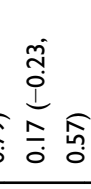 & I & $\begin{array}{l}\text { d0 } \\
0 \\
0 \\
0 \\
0 \\
0\end{array}$ & $\begin{array}{ll}0 & 1 \\
0 & 1\end{array}$ & 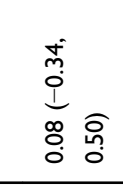 & 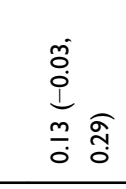 \\
\hline & & $\begin{array}{ll}\bar{y} \\
\dot{0} \\
\dot{z} \\
\dot{z}\end{array}$ & 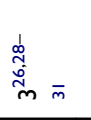 & 品 & $\underline{q}$ & 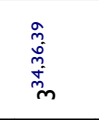 & $\stackrel{m}{-}$ & I & $\ddagger$ & I & 品 & \pm \\
\hline \multicolumn{3}{|c|}{ 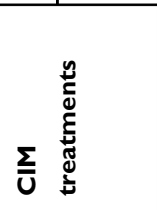 } & 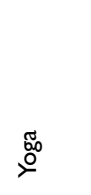 & 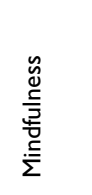 & 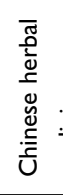 & 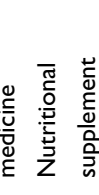 & 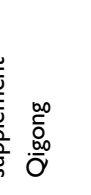 & & 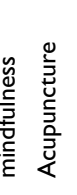 & $\begin{array}{l}: 0 \\
\text { g. } \\
\tilde{g}^{\prime} \\
\Sigma \\
\Sigma\end{array}$ & 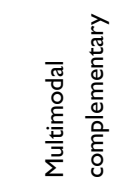 & 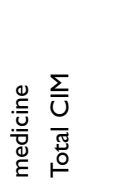 \\
\hline
\end{tabular}


A

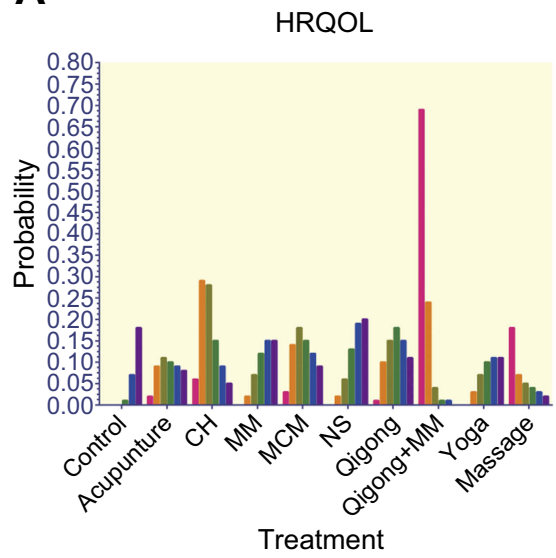

D

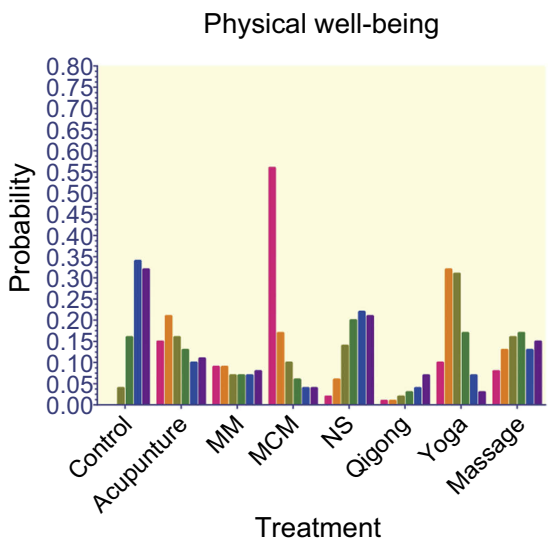

B

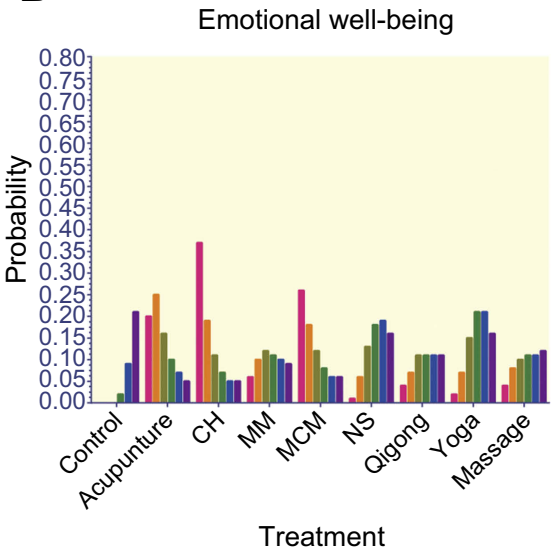

E

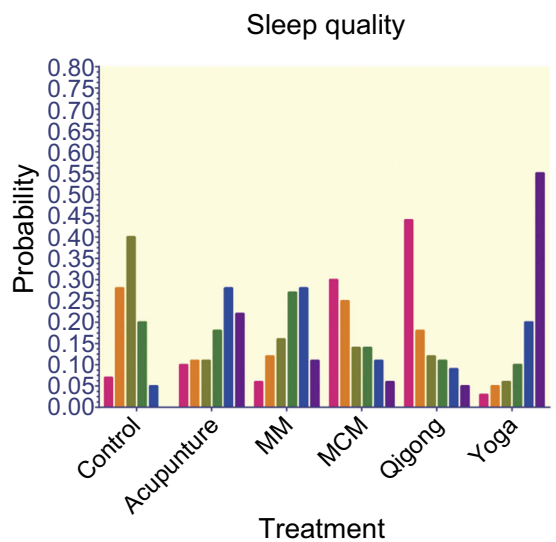

C

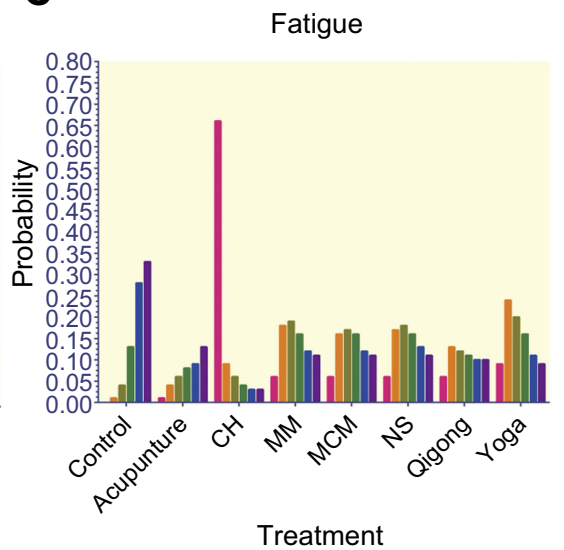

$\mathbf{F}$

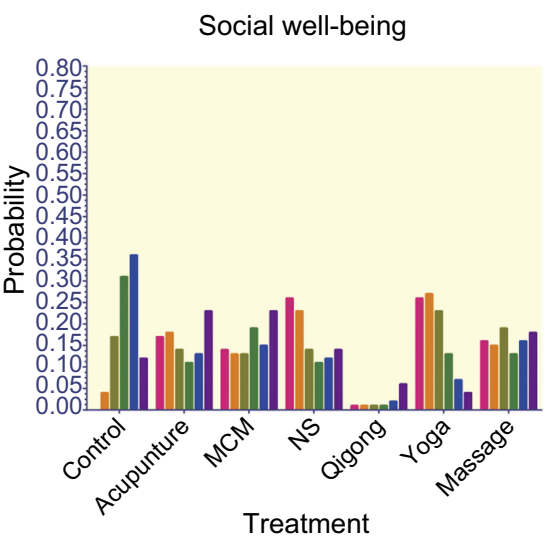

Rank 1

Rank 2

Rank 3

Rank 4

Rank 5

Rank 6

Figure 5 Ranking probability for each treatment on health-related quality of life. Rank I is best and rank 6 is worst.

Abbreviations: $\mathrm{CH}$, Chinese herbal medicine; MCM, multimodal complementary medicine; MM, mindfulness; NS, nutritional supplement.

qigong + MM (SUCRA: 0.985) was shown to be the most efficacious treatment, followed by $\mathrm{CH}$ (SUCRA: 0.865), MCM (SUCRA: 0.65), qigong (SUCRA: 0.64), NS (SUCRA: 0.59), MM (SUCRA: 0.50), acupuncture (SUCRA: 0.44), yoga (SUCRA: 0.405), massage (SUCRA: 0.365), and control (SUCRA: 0.26), which means that all the treatments are more effective than control (Figure 5A). For emotional well-being, the ranking probability was $\mathrm{CH}>$ yoga $>$ acupuncture $>\mathrm{MCM}>\mathrm{NS}$ $>\mathrm{MM}>$ massage $>$ qigong $>$ control (Figure 5B). For fatigue, the ranking probability was $\mathrm{CH}>$ yoga $>$ control $>\mathrm{MM}>\mathrm{NS}>\mathrm{MCM}>$ qigong $>$ acupuncture (Figure 5C). For physical well-being, the ranking probability was yoga $>\mathrm{MCM}>$ control $>\mathrm{NS}>$ acupuncture $>$ massage $>\mathrm{MM}>$ qigong (Figure 5D). For sleep quality, the ranking probability was control $>$ yoga $>$ qigong $>\mathrm{MM}>\mathrm{MCM}>$ acupuncture (Figure 5E). For social well-being, the ranking probability was control $>$ yoga $>$ NS $>$ MCM $>$ massage $>$ acupuncture $>$ qigong (Figure 5F).

\section{Publication bias and network coherence}

There was no evidence of publication bias, either qualitatively based on funnel-plot asymmetry (Figure 6) or quantitatively based on Begg's regression test (Figure 7) ( $P>0.05$ for all comparisons), although the number of studies included in each comparison was small. Evaluation of the Monte Carlo error suggested adequacy of convergence, which suggested good model fit.

\section{Quality of evidence}

The GRADE evidence profiles are shown in Table 3. The GRADE level of meta-analysis combining direct and indirect evidence was moderate for overall CIM interventions. Regarding each CIM intervention, the GRADE 


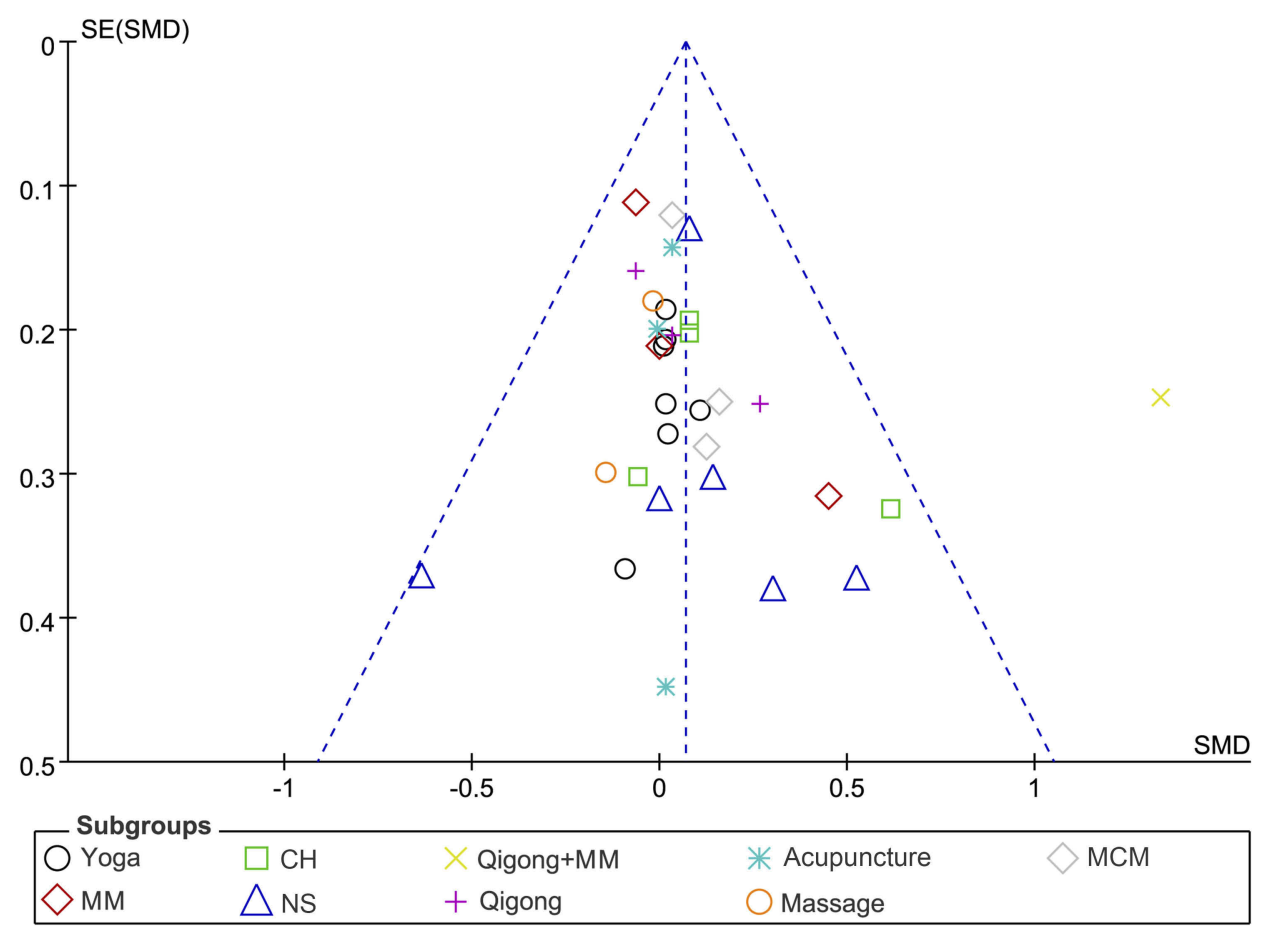

Figure 6 Funnel plot of publication bias. The dashed line represents the expected distribution of studies on the graph in the absence of publication bias. Abbreviations: $\mathrm{CH}$, Chinese herbal medicine; MCM, multimodal complementary medicine; MM, mindfulness; NS, nutritional supplement; SMD, standardized mean difference.

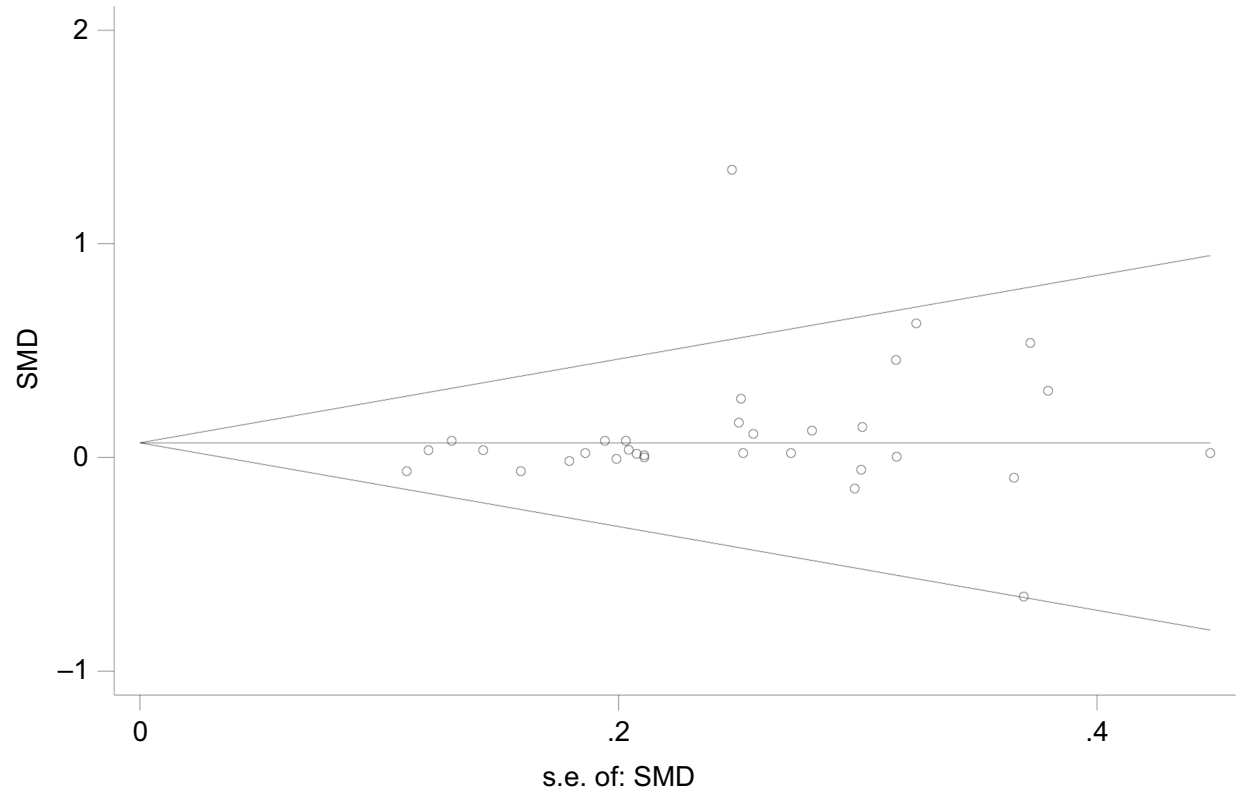

Figure 7 Begg's funnel plot with pseudo-95\% confidence limits: publication bias of complementary and integrative medicine interventions vs control. Abbreviation: SMD, standardized mean difference.

quality of evidence was moderate for NS, yoga, and $\mathrm{CH}$, while it was low for the remaining treatments.

\section{Discussion}

As a major global health problem, cancer is a terrible disease in which complications from conventional treatments may reduce the HRQOL, which in turn affects the prognosis of patients. Therefore, more and more clinicians take HRQOL into consideration when establishing a therapeutic regimen to gain an optimal response. In the present meta-analysis, we combined direct and indirect evidence from 34 RCTs in 3,010 patients with tumor to 
Table 3 Overall GRADE quality of evidence from network metaanalysis

\begin{tabular}{|l|l|}
\hline CIM & HRQOL changes from baseline \\
\hline NS & Moderate \\
Yoga & Moderate \\
CH & Moderate \\
Acupuncture & Low \\
Massage & Low \\
MM & Low \\
Qigong & Low \\
Qigong + MM & Low \\
MCM & Low \\
\hline
\end{tabular}

Abbreviations: GRADE, Grading of Recommendations Assessment, Development and Evaluation; CIM, complementary and integrative medicine; HRQOL, healthrelated quality of life; NS, Nutritional supplement; $\mathrm{CH}$, Chinese herbal medicine; MM, mindfulness; MCM, multimodal complementary medicine.

demonstrate the potential advantage of different CIM treatments on the HRQOL. The results tended to provide moderate-quality or low-quality evidence for moderate beneficial effects of CIM interventions on the HRQOL of cancer patients. As a whole, the change in HRQOL scores was statistically significant between CIM treatment (mean range from 82.5 to 87.0 ) and control (mean range from 80.4 to 81.8 ) groups. Direct meta-analysis for total effect also showed the clearly superior efficacy of CIM treatments, although subgroup analysis demonstrated that only $\mathrm{CH}$ and qigong + $\mathrm{MM}$ were significantly favored more than usual care.

In this study, yoga was the most studied intervention, with eight trials included in the analysis, followed by $\mathrm{NS}, \mathrm{CH}$, and acupuncture. When we searched PubMed briefly with random words we found that the most papers had been published on NS, followed by acupuncture, $\mathrm{CH}$, and yoga, which indicated that studies on acupuncture and $\mathrm{CH}$ may focus more on the unambiguous diseases rather than functional discomfort such as HRQOL. However, it may also be due to the complexity of acupuncture and $\mathrm{CH}$, for which it is difficult to make a blank control, an essential requirement for RCTs. ${ }^{62-64}$ Therefore, more RCTs on acupuncture and $\mathrm{CH}$ are needed to explore their effects on HRQOL of cancer patients, since they have been elaborately studied in the fields of specific diseases such as cancers. Nearly half of the included studies were conducted in the USA, which may be related to its highly developed medical research. This may influence the conclusions of our study and the multiplicity of study locations serves to increase the level of confidence in our findings.
In 2012, about 14.1 million new cases of cancer were diagnosed worldwide, with the most common types being lung (13\%), breast $(12 \%)$, and colorectal cancer $(10 \%){ }^{65}$ In our analysis, breast cancer was the most studied cancer and most of the participators $(92 \%)$ were female, with only one study focusing on the most widespread cancer, ie, lung cancer. Only $8 \%$ of the enrolled participants were male, which may reduce the credibility of our conclusions and make it difficult to recommend such CIM interventions among male cancer patients. Therefore, more high-quality RCTs, in greater detail and focusing on various cancers among both female and male patients, are needed and will prove valuable.

Although tests showed the clearly superior efficacy of CIM treatments in improving HRQOL, yoga seems to aggravate sleep quality (SMD $-0.81[-1.18,-0.08]$ ). However, there was only one study reporting the sleep scores after treatment with yoga, so more research is needed to clarify the effects of yoga on sleep quality with more certainty.

Traditional Chinese medicine (TCM) has been long practiced and is becoming ever more widely recognized as providing curative and/or healing treatments for a number of diseases and physiological conditions. ${ }^{66} \mathrm{CH}$, acupuncture, and moxibustion are among the most popular types of TCM. CH showed a significantly superior relative effect on HRQOL, emotional well-being, and fatigue. $\mathrm{CH}$ is complicated and variable since it often employs combined prescriptions of multiple herbs for disease treatment. ${ }^{64}$ Such complexity and variability are based on an empirical set of principles that is referred to as monarch, minister, assistant, and guide. ${ }^{67}$ Therefore, only a small number of RCTs on $\mathrm{CH}$ have been conducted, most of which are of poor methodological quality owing to difficulties in the design and implementation of placebo-blinded trials. ${ }^{68}$ Modernization of $\mathrm{CH}$, such as pharmacological studies including chemistry-focused, target-directed, and systems-biology-based studies, may promote its development. Acupuncture, however, in this analysis, did not show superiority in improving HRQOL. In accord with previous studies, ${ }^{69}$ yoga was found to play an important role in both emotional and physical well-being.

Although all included studies were RCTs without obvious risks of bias, limitations are present and should be accounted for when interpreting the study's findings. First, only 34 RCTs were included in the present study according to the selected criteria and no more than eight trials were conducted for each CIM intervention. The 
small sample size limited statistical power and study generalizability, meaning that the actual effects of CIMs may be small, although they showed superior relative effects from the existing data. Individual patient data and more detailed subgroups would have enabled us to provide more detailed insights. So, larger and more diverse samples are needed to calculate the best intervention for the exact tumor type and even the exact domains, such as emotional well-being or sleep quality, to remove the potentially confounding influence of such differences. On the other hand, other psychosocial support services such as music and art therapy or psychological counseling may also make sense, although they are excluded from the present study. The HRQOL of tumor patients is often complex and difficult to resolve, and a consistently effective CIM treatment is still lacking, making it important to examine this in future research. Second, although we have tried to figure out which intervention may be best for HRQOL by ranking the probability for each treatment using ADDIS, there is still a lack of clinical trials comparing the different efficacy of different CIMs. In addition, research focusing more on male cancer patients may make the recommended CIMs more convincing for all cancer patients. More tools which are commonly used in integrative oncology research, such as Measure Yourself Concerns and Wellbeing $(\mathrm{MYCaW})^{70}$ and the Edmonton Symptom Assessment Scale (ESAS), ${ }^{71}$ may be used in future research.

\section{Conclusion}

This systematic review provides a comprehensive overview of the relationship between different CIM interventions and the HRQOL of tumor patients. The results demonstrated clearly superior efficacy of CIM treatments in improving HRQOL, and different CIM interventions may play different roles in HRQOL such as emotional and physical well-being. More studies, especially focusing on male cancer patients, are needed to increase the confidence levels of our findings.

\section{Data sharing}

Data are available from the corresponding authors at cbb8202@126.com (BBC) or changquanling@smmu.edu. cn (CQL).

\section{Acknowledgments}

We thank Yanyan Wei for valuable advice and helpful discussions. This research was funded by grants from the
National Natural Science Foundation of China (numbers 81430101 and 81774077).

\section{Author contributions}

All authors contributed toward data analysis, drafting and critically revising the paper, gave final approval of the version to be published, and agree to be accountable for all aspects of the work.

\section{Disclosure}

The authors report no conflicts of interest in this work.

\section{References}

1. Torre LA, Bray F, Siegel RL, Ferlay J, Lortet-Tieulent J, Jemal A. Global cancer statistics, 2012. CA Cancer J Clin. 2015;65(2):87-108. doi:10.3322/caac. 21262

2. Prigerson HG, Bao Y, Shah MA, et al. Chemotherapy Use, Performance Status, and Quality of Life at the End of Life. JAMA Oncol. 2015;1(6):778-784. doi:10.1001/jamaoncol.2015.2378

3. Kovic B, Guyatt G, Brundage M, Thabane L, Bhatnagar N, Xie F. Association between progression-free survival and health-related quality of life in oncology: a systematic review protocol. $B M J$ Open. 2016;6(9):e012909. doi:10.1136/bmjopen-2016-012909

4. Zhou ZY, Xu L, Li HG, et al. Chemotherapy in conjunction with traditional Chinese medicine for survival of elderly patients with advanced non-small-cell lung cancer: protocol for a randomized double-blind controlled trial. J Integr Med. 2014;12(3):175-181. doi:10.1016/S2095-4964(14)60028-5

5. Leong M, Smith TJ, Rowland-Seymour A. Complementary and integrative medicine for older adults in palliative care. Clin Geriatr Med. 2015;31(2):177-191. doi:10.1016/j.cger.2015.01.004

6. Frenkel M, Cohen L. Incorporating complementary and integrative medicine in a comprehensive cancer center. Hematol Oncol Clin North Am. 2008;22(4):727-736, ix. doi:10.1016/j.hoc.2008.04.002

7. Greenlee H, DuPont-Reyes MJ, Balneaves LG, et al. Clinical practice guidelines on the evidence-based use of integrative therapies during and after breast cancer treatment. CA Cancer J Clin. 2017;67 (3):194-232. doi:10.3322/caac.21397

8. Ling CQ, Fan J, Lin HS, et al. Clinical practice guidelines for the treatment of primary liver cancer with integrative traditional Chinese and Western medicine. $J$ Integr Med. 2018;16(4):236-248. doi:10.1016/j.joim.2018.05.002

9. Hutton B, Salanti G, Caldwell DM, et al. The PRISMA extension statement for reporting of systematic reviews incorporating network meta-analyses of health care interventions: checklist and explanations. Ann Intern Med. 2015;162(11):777-784. doi:10.7326/ M14-2385

10. Malone DC, Berg NS, Claxton K, et al. International society for pharmacoeconomics and outcomes research comments on the American Society of Clinical Oncology value framework. $J$ Clin Oncol. 2016;34(24):2936-2937. doi:10.1200/JCO.2015.64.4328

11. Hoaglin DC, Hawkins N, Jansen JP, et al. Conducting indirect-treatment-comparison and network-meta-analysis studies: report of the ISPOR Task Force on Indirect Treatment Comparisons Good Research Practices: part 2. Value Health. 2011;14(4):429-437. doi:10.1016/j.jval.2011.01.011

12. Puhan MA, Schunemann HJ, Murad MH, et al. A GRADE Working Group approach for rating the quality of treatment effect estimates from network meta-analysis. BMJ. 2014;349:g5630. doi:10.1136/bmj.g5630 
13. Boers-Doets CB, Gelderblom H, Lacouture ME, et al. Translation and linguistic validation of the FACT-EGFRI-18 quality of life instrument from English into Dutch. Eur J Oncol Nurs. 2013;17 (6):802-807. doi:10.1016/j.ejon.2013.03.004

14. Webster K, Cella D, Yost K. The Functional Assessment of Chronic Illness Therapy (FACIT) measurement system: properties, applications, and interpretation. Health Qual Life Outcomes. 2003;1:79. doi:10.1186/1477-7525-1-79

15. Sailors MH, Bodurka DC, Gning I, et al. Validating the M. D. Anderson Symptom Inventory (MDASI) for use in patients with ovarian cancer. Gynecol Oncol. 2013;130(2):323-328. doi:10.1016/j.ygyno.2013.05.009

16. Antonescu I, Carli F, Mayo NE, Feldman LS. Validation of the SF-36 as a measure of postoperative recovery after colorectal surgery. Surg Endosc. 2014;28(11):3168-3178. doi:10.1007/s00464-014-3577-8

17. Zhang L, Dai Z, Cheng S, et al. Validation of EORTC IN-PATSAT32 for Chinese cancer patients. Support Care Cancer. 2015;23 (9):2721-2730. doi:10.1007/s00520-015-2636-6

18. Higgins JP, Altman DG, Gotzsche PC, et al. The Cochrane Collaboration's tool for assessing risk of bias in randomised trials BMJ. 2011;343:d5928. doi:10.1136/bmj.d5928

19. Khera R, Murad MH, Chandar AK, et al. Association of pharmacological treatments for obesity with weight loss and adverse events: a systematic review and meta-analysis. JAMA. 2016;315(22):2424-2434. doi:10.1001/ jama.2016.7602

20. DerSimonian R, Laird N. Meta-analysis in clinical trials. Control Clin Trials. 1986;7(3):177-188.

21. Higgins JP, Thompson SG, Deeks JJ, Altman DG. Measuring inconsistency in meta-analyses. BMJ. 2003;327(7414):557-560. doi:10.1136/ bmj.327.7414.557

22. Hartung J, Knapp G. A refined method for the meta-analysis of controlled clinical trials with binary outcome. Stat Med. 2001;20(24):3875-3889.

23. Chaimani A, Higgins JP, Mavridis D, Spyridonos P, Salanti G. Graphical tools for network meta-analysis in STATA. PLoS One. 2013;8(10):e76654. doi:10.1371/journal.pone.0076654

24. Zeng XT, Zhang $\mathrm{C}$, Du L. Using ADDIS Software for Implementation Network Meta-Analysis. Chin J Evid-Based Med. 2013,13(12):1508-1515. doi:10.7507/1672-2531.20130257.

25. Dias S, Welton N, Sutton A, Ades A. NICE DSU Technical Support Document 2: A Generalised Linear Modelling Framework for Pairwise and Network Meta-Analysis of Randomised Controlled Trials. London: National Institute for Health and Care Excellence (NICE); 2011.

26. Cramer H, Pokhrel B, Gass F, et al. Hatha yoga for patients with colorectal cancer: a randomized controlled mixed-methods study. $J$ Altern Complement Med. 2014;20(5):A52-A53. doi:10.1089/acm.2014.5137. abstract

27. Harder H, Langridge C, Solis-Trapala I, et al. Post-operative exercises after breast cancer surgery: results of a RCT evaluating standard care versus standard care plus additional yoga exercise. Eur $J$ Integr Med. 2015;7(3):202-210. doi:10.1016/j.eujim.2015.02.002

28. Siedentopf F, Utz-Billing I, Gairing S, Schoenegg W, Kentenich H, Kollak I. Yoga for patients with early breast cancer and its impact on quality of life - a randomized controlled trial. Geburtshilfe Frauenheilkd. 2013;73(4):311-317. doi:10.1055/s-0032-1328438

29. Littman AJ, Bertram LC, Ceballos R, et al. Randomized controlled pilot trial of yoga in overweight and obese breast cancer survivors: effects on quality of life and anthropometric measures. Support Care Cancer. 2012;20(2):267-277. doi:10.1007/s00520-010-1066-8

30. Chandwani KD, Thornton B, Perkins GH, et al. Yoga improves quality of life and benefit finding in women undergoing radiotherapy for breast cancer. J Soc Integr Oncol. 2010;8(2):43-55.

31. Moadel AB, Shah C, Wylie-Rosett J, et al. Randomized controlled trial of yoga among a multiethnic sample of breast cancer patients: effects on quality of life. J Clin Oncol. 2007;25(28):4387-4395. doi:10.1200/JCO.2006.06.6027
32. Pruthi S, Stan DL, Jenkins SM, et al. A randomized controlled pilot study assessing feasibility and impact of yoga practice on quality of life, mood, and perceived stress in women with newly diagnosed breast cancer. Global Adv Health Med. 2012;1(5):30-35. doi:10.7453/gahmj.2012.1.5.010

33. Ben-Josef AM, Chen J, Wileyto P, et al. Effect of eischens yoga during radiation therapy on prostate cancer patient symptoms and quality of life: a randomized phase II trial. Int J Radiat Oncol Biol Phys. 2017;98(5):1036-1044. doi:10.1016/j.ijrobp.2017.03.043

34. Lesser GJ, Case D, Stark N, et al. A randomized, double-blind, placebo-controlled study of oral coenzyme Q10 to relieve self-reported treatment-related fatigue in newly diagnosed patients with breast cancer. $J$ Support Oncol. 2013;11(1):31-42. doi:10.1016/j.suponc.2012.03.003

35. Lustberg MB, Orchard TS, Reinbolt R, et al. Randomized placebo-controlled pilot trial of omega 3 fatty acids for prevention of aromatase inhibitor-induced musculoskeletal pain. Breast Cancer Res Treat. 2018;167(3):709-718. doi:10.1007/s10549-017-4559-Z

36. Noguchi N, Maruyama I, Yamada A. The influence of chlorella and its hot water extract supplementation on quality of life in patients with breast cancer. Evid Based Complement Alternat Med. 2014;2014:704619. doi:10.1155/2014/105186

37. Norman K, Stubler D, Baier P, et al. Effects of creatine supplementation on nutritional status, muscle function and quality of life in patients with colorectal cancer-a double blind randomised controlled trial. Clin Nutr. 2006;25(4):596-605. doi:10.1016/j. clnu.2006.01.014

38. Can G, Topuz E, Derin D, Durna Z, Aydiner A. Effect of kefir on the quality of life of patients being treated for colorectal cancer. Oncol Nurs Forum. 2009;36(6):E335-E342. doi:10.1188/09.ONF.E335-E342

39. Cruciani RA, Dvorkin E, Homel P, et al. L-carnitine supplementation in patients with advanced cancer and carnitine deficiency: a double-blind, placebo-controlled study. J Pain Symptom Manage. 2009;37(4):622-631. doi:10.1016/j.jpainsymman.2008.03.021

40. Han Y, Wang $\mathrm{H}, \mathrm{Xu} \mathrm{W}$, et al. Chinese herbal medicine as maintenance therapy for improving the quality of life for advanced non-small cell lung cancer patients. Complement Ther Med. 2016;24:81-89. doi:10.1016/j.ctim.2015.12.008

41. Marx W, McCarthy AL, Ried K, et al. The effect of a standardized ginger extract on chemotherapy-induced nausea-related quality of life in patients undergoing moderately or highly emetogenic chemotherapy: a double blind, randomized, placebo controlled trial. Nutrients. 2017;9(8). doi:10.3390/nu9080867

42. Tian HQ, Li HL, Wang B, et al. Treatment of middle/late stage primary hepatic carcinoma by Chinese medicine comprehensive therapy: a prospective randomized controlled study. Chin J Integr Med. 2010;16(2):102-108. doi:10.1007/s11655-010-0102-3

43. Jeong JS, Ryu BH, Kim JS, Park JW, Choi WC, Yoon SW. Bojungikki-tang for cancer-related fatigue: a pilot randomized clinical trial. Integr Cancer Ther. 2010;9(4):331-338. doi:10.1177/ 1534735410383170

44. Johnston MF, Hays RD, Subramanian SK, et al. Patient education integrated with acupuncture for relief of cancer-related fatigue randomized controlled feasibility study. BMC Complement Altern Med. 2011;11:49. doi:10.1186/1472-6882-11-49

45. Smith C, Carmady B, Thornton C, Perz J, Ussher JM. The effect of acupuncture on post-cancer fatigue and well-being for women recovering from breast cancer: a pilot randomised controlled trial. Acupunct Med. 2013;31(1):9-15. doi:10.1136/acupmed-2012-010228

46. Zick SM, Sen A, Wyatt GK, Murphy SL, Arnedt JT, Harris RE. Investigation of 2 types of self-administered acupressure for persistent cancer-related fatigue in breast cancer survivors: a randomized clinical trial. JAMA Oncol. 2016;2(11):1470-1476. doi:10.1001/ jamaoncol.2016.1867 
47. Deng G, Chan Y, Sjoberg D, et al. Acupuncture for the treatment of post-chemotherapy chronic fatigue: a randomized, blinded, sham-controlled trial. Support Care Cancer. 2013;21(6):1735-1741. doi:10.1007/s00520-013-1720-z

48. Spahn G, Choi KE, Kennemann C, et al. Can a multimodal mind-body program enhance the treatment effects of physical activity in breast cancer survivors with chronic tumor-associated fatigue? A randomized controlled trial. Integr Cancer Ther. 2013;12 (4):291-300. doi:10.1177/1534735413492727

49. Domnick M, Domnick M, Wiebelitz KR, Beer AM. Evaluation of the effectiveness of a multimodal complementary medicine program for improving the quality of life of cancer patients during adjuvant radiotherapy and/or chemotherapy or outpatient aftercare. Oncology. 2017;93(2):83-91. doi:10.1159/000468939

50. Witt CM, Ausserer O, Baier S, et al. Effectiveness of an additional individualized multi-component complementary medicine treatment on health-related quality of life in breast cancer patients: a pragmatic randomized trial. Breast Cancer Res Treat. 2015;149(2):449-460. doi:10.1007/s10549-014-3249-3

51. Chen Z, Meng Z, Milbury K, et al. Qigong improves quality of life in women undergoing radiotherapy for breast cancer: results of a randomized controlled trial. Cancer. 2013;119(9):1690-1698. doi:10.1002/cncr.27904

52. Liu P, You J, Loo WTY, et al. The efficacy of Guolin-Qigong on the body-mind health of Chinese women with breast cancer: a randomized controlled trial. Qual Life Res. 2017;26 (9):2321-2331. doi:10.1007/s11136-017-1576-7

53. Loh SY, Lee SY, Murray L. The Kuala Lumpur Qigong trial for women in the cancer survivorship phase-efficacy of a three-arm RCT to improve QOL. Asian Pac J Cancer Prev. 2014;15(19):8127-8134.

54. Blaes A, Fenner D, Bachanova V, et al. Mindfulness-Based Cancer Recovery in Survivors Recovering from Chemotherapy and Radiation. J Community Support Oncol. 2016;14(8):351-358. doi: $10.12788 /$ jcso.0244

55. Milbury K, Chaoul A, Biegler K, et al. Tibetan sound meditation for cognitive dysfunction: results of a randomized controlled pilot trial. Psycho-Oncology. 2013;22(10):2354-2363. doi:10.1002/pon.3296

56. Reich RR, Lengacher CA, Alinat CB, et al. Mindfulness-based stress reduction in post-treatment breast cancer patients: immediate and sustained effects across multiple symptom clusters. J Pain Symptom Manage. 2017;53(1):85-95. doi:10.1016/j.jpainsymman.2016.08.005

57. Sharp DM, Walker MB, Chaturvedi A, et al. A randomised, controlled trial of the psychological effects of reflexology in early breast cancer. Eur $J$ Cancer. 2010;46(2):312-322. doi:10.1016/j.ejca.2009.10.006

58. Judson PL, Dickson EL, Argenta PA, et al. A prospective, randomized trial of integrative medicine for women with ovarian cancer. Gynecol Oncol. 2011;123(2):346-350. doi:10.1016/j.ygyno.2011.07.099

59. Oh B, Butow PN, Mullan BA, et al. Effect of medical Qigong on cognitive function, quality of life, and a biomarker of inflammation in cancer patients: a randomized controlled trial. Support Care Cancer. 2012;20(6):1235-1242. doi:10.1007/s00520-011-1209-6
60. Daukantaite D, Tellhed U, Maddux RE, Svensson T, Melander O. Five-week yin yoga-based interventions decreased plasma adrenomedullin and increased psychological health in stressed adults: A randomized controlled trial. PLoS One. 2018;13(7):e0200518. doi:10.1371/journal.pone.0200518

61. Salanti G, Ades AE, Ioannidis JP. Graphical methods and numerical summaries for presenting results from multiple-treatment meta-analysis: an overview and tutorial. J Clin Epidemiol. 2011;64 (2):163-171. doi:10.1016/j.jclinepi.2010.03.016

62. Liu T. Route of placebo administration: robust placebo effects in laboratory and clinical settings. Neurosci Biobehav Rev. 2017;83:451-457. doi:10.1016/j.neubiorev.2017.09.018

63. Han JS, Ho YS. Global trends and performances of acupuncture research. Neurosci Biobehav Rev. 2011;35(3):680-687. doi:10.1016/ j.neubiorev.2010.08.006

64. Wang D, Calabrese EJ, Lian B, Lin Z, Calabrese V. Hormesis as a mechanistic approach to understanding herbal treatments in traditional Chinese medicine. Pharmacol Ther. 2018;184:42-50. doi:10.1016/j.pharmthera.2017.10.013

65. Steffens D, Beckenkamp PR, Hancock M, Solomon M, Young J. Preoperative exercise halves the postoperative complication rate in patients with lung cancer: a systematic review of the effect of exercise on complications, length of stay and quality of life in patients with cancer. Br J Sports Med. 2018;52(5):344. doi:10.1136/bjsports2017-098032

66. Thirthalli J, Zhou L, Kumar K, et al. Traditional, complementary, and alternative medicine approaches to mental health care and psychological wellbeing in India and China. Lancet Psychiatry. 2016;3 (7):660-672. doi:10.1016/S2215-0366(16)30025-6

67. Chen X, Pei L, Lu J. Filling the gap between traditional Chinese medicine and modern medicine, are we heading to the right direction? Complement Ther Med. 2013;21(3):272-275. doi:10.1016/j.ctim.2013.01.001

68. Tang JL. Research priorities in traditional Chinese medicine. BMJ. 2006;333(7564):391-394. doi:10.1136/bmj.333.7564.391

69. Pascoe MC, Thompson DR, Ski CF. Yoga, mindfulness-based stress reduction and stress-related physiological measures: A meta-analysis. Psychoneuroendocrinology. 2017;86:152-168. doi:10.1016/j.psyneuen. 2017.08.008

70. Frenkel M, Cohen L, Peterson N, et al. Integrative medicine consultation service in a comprehensive cancer center: findings and outcomes. Integr Cancer Ther. 2010;9(3):276-283. doi:10.1177/ 1534735410378663

71. Sharabi IS, Levin A, Schiff E, et al. Quality of life-related outcomes from a patient-tailored integrative medicine program: experience of Russian-speaking patients with cancer in Israel. Support Care Cancer. 2016;24(10):1-5. doi:10.1007/s00520-016-3274-3

\section{Publish your work in this journal}

Cancer Management and Research is an international, peer-reviewed open access journal focusing on cancer research and the optimal use of preventative and integrated treatment interventions to achieve improved outcomes, enhanced survival and quality of life for the cancer patient.
The manuscript management system is completely online and includes a very quick and fair peer-review system, which is all easy to use. Visit http://www.dovepress.com/testimonials.php to read real quotes from published authors. 